\title{
Suppressive effect of an analog of the antimicrobial peptide of LL-37 on colon cancer cells via exosome-encapsulated miRNAs
}

\author{
MIWA HAYASHI, KENGO KURODA, KOHEI IHARA, TAKAHIRO IWAYA and EMIKO ISOGAI \\ Laboratory of Animal Microbiology, Graduate School of Agricultural Science, \\ Tohoku University, Sendai, Miyagi 980-0845, Japan
}

Received March 8, 2018; Accepted June 21, 2018

DOI: $10.3892 / \mathrm{ijmm} .2018 .3875$

\begin{abstract}
Antimicrobial peptides (AMPs) are multifunctional factors with an important role in the innate immune system. Our previous studies revealed that the human cathelicidin LL-37 and its analog, FF/CAP18, limit the proliferation of colon cancer cell lines. In the present study, the exosomes released by HCT116 cells treated with FF/CAP18 were analyzed. After the treatment, exosomes were isolated from the culture supernatant by ultrafiltration and using the miRCURY ${ }^{\mathrm{TM}}$ Exosome Isolation Kit. Membrane vesicles 40-100-nm expressing CD63 and CD81 were identified before and after FF/CAP18 treatment. Exosome concentration in the culture supernatant was increased after treatment with FF/CAP18. Exosomes formed in HCT116 cells treated with FF/CAP18 induced growth suppression of the cells in a dose-dependent manner. By contrast, the exosomes formed in non-treated HCT116 cells did not affect cell viability. Microarray analysis of miRNA expression indicated that FF/CAP18 treatment induced increases in the expression of three miRNAs (miR-584-5p, miR-1202 and miR-3162-5p) in both HCT116 cells and exosomes. These results suggest that FF/CAP18 treatment increases exosome formation, and that exosome-encapsulated miRNAs suppress HCT116 cell proliferation. Exosomal miRNAs are considered to be involved in the dissemination of cell signals to control local cellular microenvironments. The present findings suggest that FF/CAP18 regulates cancer growth by modulating cell-to-cell communication. AMPs localize in the cytoplasm of cancer cells and enhance the expression of growth-suppressing miRNAs. These miRNAs are also transported to other cancer cells via exosomes. Therefore, transportation of these miRNAs has the potential to suppress cancer growth. AMPs exert their
\end{abstract}

Correspondence to: Professor Emiko Isogai, Laboratory of Animal Microbiology, Graduate School of Agricultural Science, Tohoku University, 468-1 Aoba, Aramaki, Aoba-ku, Sendai, Miyagi 980-0845, Japan

E-mail: homeiso2006@yahoo.co.jp

Key words: exosome, HCT116 cells, antimicrobial peptides, LL-37, miR-584-5p, miR-1202, miR-3162-5p effects directly by targeting cancer cells and indirectly via exosomes.

\section{Introduction}

Antimicrobial peptides (AMPs) in the cathelicidin family are multifunctional molecules found in all mammalian species (1). Human cathelicidin hCAP18/LL-37 was reported to prevent infections via the innate immune system (2). LL-37 is widely expressed in several types of cells and organs, including neutrophils, skin, epithelial cells of the airways and colon epithelium (3-6), and is considered to suppress cancers, such as colon cancer (7).

Colon cancer is one of the most commonly diagnosed malignant tumors in men and women. A total of 1.4 million colon cancer cases were estimated and $\sim 700,000$ deaths were reported in 2012 worldwide (8). There are six types of standard treatment for colon cancer, including surgery and chemotherapy (9). However, the effectiveness of chemotherapeutic agents differs among patients. Therefore, novel biological agents and treatment approaches for colon cancer are needed. The present study focused on AMPs as one of the possible biological therapeutic agents.

Our group previously reported that a 27-residue analog of the LL-37 peptide, FF/CAP18, induced apoptosis in SAS-H1 oral squamous cell carcinoma cells (10) and the colon carcinoma-derived cell line HCT116 (11). FF/CAP18 upregulates miR-663a in HCT116 cells. MicroRNAs (miRNAs) regulate the growth of HCT116 cells by targeting the CXCR4-p21 pathway (12).

Exosomes are small extracellular membrane vesicles (30-100 nm) secreted by numerous types of cells, including cancer cells. Exosomes contain protein and nucleic acids, such as miRNAs, and act as important mediators of intercellular communication $(13,14)$. The association between exosomes and cancer cells has been widely investigated. Specific miRNAs transported in cancer-secreted exosomes have been found to promote metastasis (15) and are potential biomarkers of cancer (16).

Exosomal miRNAs play critical roles in regulating cancer development. miRNAs are small non-coding RNAs that control gene expression by binding to the 3 '-untranslated region of their target mRNA (17). Alterations of miRNAs are involved in the initiation and progression of cancer (18), 
whereas miRNAs may act as tumor suppressors as well as oncogenes (19). miRNAs are present in cancer cells and extracellularly in exosomes $(13,20)$. However, cell-to-cell communication in colon cancer cells following treatment with AMPs has not yet been elucidated. Therefore, the aim of the present study was to focus on the release of exosomes from HCT116 cells treated with FF/CAP18 and cell-to-cell communication following treatment with AMPs. The quality and quantity of exosomes secreted by colon cancer HCT116 cells and upregulated miRNAs in both the cells and exosomes were analyzed following treatment with FF/CAP18.

\section{Materials and methods}

Cell culture. Cells from the HCT116 human colon carcinoma-derived cell line were provided by Dr Bert Vogelstein (The Johns Hopkins University, Baltimore, MD, USA). The cells were cultured in Dulbecco's modified Eagle's medium (DMEM; Nacalai Tesque, Kyoto, Japan) containing 10\% fetal bovine serum (Invitrogen; Thermo Fisher Scientific, Inc., Carlsbad, CA, USA) and 5\% antibiotic/antimycotic mixed stock solution (Nacalai Tesque) at $37^{\circ} \mathrm{C}$ and $5 \% \mathrm{CO}_{2}$. Cell passage and maintenance were performed as previously described (12).

Peptides. The primary amino acid structure of the original LL-37 is LLGDFFRKSKEKIGKEFKRIVQRIKDFLRNL VPRTES. hCAP is a 27-mer peptide that lacks the first and last 5 amino acids of LL-37. FF/CAP18 (FRKSKEKIGKFF KRIVQRIFDFLRNLV) was designed by replacing a glutamic acid residue and lysine residue with phenylalanine of hCAP18 to enhance its antimicrobial and anticancer properties (11).

Exosome isolation. HCT116 cells were seeded at a density of $1.2 \times 10^{6}$ cells $/ \mathrm{ml}$ in a $100-\mathrm{mm}$ dish and incubated for 2 days. The cells were washed with phosphate-buffered saline and cultivated in DMEM containing 10\% exosome-depleted fetal bovine serum Media Supplement (System Biosciences, Palo Alto, CA, USA) and 5\% antibiotic/antimycotic mixed stock solution (Nacalai Tesque). After $48 \mathrm{~h}$, the medium was harvested to collect exosomes. Exosomes were isolated by ultrafiltration using a Vivaspin 20-100K (GE Healthcare, Little Chalfont, UK) and miRCURY ${ }^{\mathrm{TM}}$ Exosome Isolation Kit-Cells, Urine and CSF (Exiqon, Vedbaek, Denmark). The culture medium was centrifuged at $300 \mathrm{xg}$ for $5 \mathrm{~min}$ and at $1,200 \mathrm{xg}$ for $20 \mathrm{~min}$ to remove cells and debris. The supernatant $(20 \mathrm{ml})$ was ultrafiltered and condensed to $1 \mathrm{ml}$ using the Vivaspin $20-100 \mathrm{~K}$ to concentrate the exosomes. Exosome isolation was performed using a miRCURY ${ }^{\mathrm{TM}}$ Exosome Isolation Kit according to the manufacturer's instructions (Exiqon). The protein levels of exosomes resuspended in $100 \mu 1$ of resuspension buffer (Exiqon) were quantified using the DC protein assay (Bio-Rad Laboratories, Inc., Hercules, CA, USA). After confirmation by transmission electron microscopy (TEM), a combination method was used to isolate exosomes from FF/CAP18-treated cells.

$T E M$. To examine the quality of isolated exosomes, the size, size distribution and morphology of non-treated HCT116 cells were evaluated. A total of $5 \mu 1$ of each exosome sample $(n=3)$ was applied to a 400-mesh copper grid for $10 \mathrm{~min}$ prior to negative staining with $10 \mu \mathrm{l}$ of $2 \%$ phosphotungstic acid for $10 \mathrm{~min}$. The grid was washed with $10 \mu \mathrm{l}$ of distilled water, dried, and viewed by TEM using a model H-7650 microscope (Hitachi, Tokyo, Japan).

Western blot analysis. Cells and exosomes were homogenized in lysis buffer [1 M Tris- $\mathrm{HCl}(\mathrm{pH} 7.4), 3 \mathrm{M} \mathrm{NaCl}, 1 \%$ Triton X-100, $6 \mathrm{mM}$ sodium deoxycholate, and $0.5 \%$ protease inhibitor; Nacalai Tesque] by ultrasonic fragmentation as previously described (12). Protein concentration was measured using the DC protein assay kit. Cell lysates $(20 \mu \mathrm{g})$ and exosome lysates $(10 \mu \mathrm{g})$ were electrophoresed on $5-20 \%$ polyacrylamide gels (ATTO, Tokyo, Japan) and transferred to Immobilon-P membranes (Merck Millipore, Darmstadt, Germany). The membranes were blocked with phosphate-buffered saline containing 3\% skimmed milk and $0.05 \%$ Tween-20, and then incubated with primary mouse monoclonal antibodies against CD63 (1:1,000; 10628D; Invitrogen; Thermo Fisher Scientific, Inc.), CD81 (1:1,000; 10630D; Invitrogen; Thermo Fisher Scientific, Inc.) and $\alpha$-tubulin (1:1,000; sc-32293; Santa Cruz Biotechnology, Inc., Dallas, TX, USA). After washing, the membranes were incubated with sheep anti-mouse IgG conjugated to horseradish peroxidase $(1: 2,000$; GE Healthcare) as the secondary antibody. Signals were detected using a SuperSignal $^{\mathrm{TM}}$ West Femto Maximum Sensitivity Substrate Trial Kit (Thermo Fisher Scientific, Inc., Waltham, MA, USA), and images were obtained with the LumiCube device (Liponics, Inc., Tokyo, Japan) and analyzed with Just TLC software (Sweday, Sodra Sandby, Sweden).

Localization of FAM-FF/CAP18. Synthetic FF/CAP18 was conjugated with 5-carboxyfluorescein (FAM; Scrum Co. Ltd., Tokyo, Japan). HCT116 cells were examined at 1 and $6 \mathrm{~h}$ after treatment with FAM-FF/CAP18 $(40 \mu \mathrm{g} / \mathrm{ml})$. The nuclei were visualized by staining with $1 \mu \mathrm{g} / \mathrm{ml}$ 4',6-diamidino-2-phenylindole (Dojindo Laboratories, Kumamoto, Japan) $6 \mathrm{~h}$ after treatment with FAM-FF/CAP18. The mitochondria were also visualized by staining with $200 \mathrm{nM}$ MitoRed (Dojindo Laboratories). The staining was visualized using a BZ-8100 fluorescence microscope (Keyence, Tokyo, Japan). Each experiment was conducted in triplicate.

Detection of apoptosis using Annexin V-7-amino-actinomycin $D(7-A A D)$. The rate of cell apoptosis following treatment with FF/CAP18 was measured using the Muse ${ }^{\circledR}$ Annexin V and Dead Cell assay kit (Merck Millipore). This assay is based on the appearance of phosphatidylserine on the surface of cells early during apoptosis, which can bind Annexin V. In the late phase of apoptosis or necrosis, phosphatidylserine on the cell surface binds to Annexin V and DNA binds to 7-AAD, which is a fluorescent intercalator of GC regions. The combination of Annexin V and 7-AAD is used to detect apoptotic cells and distinguish between apoptotic phases. HCT116 cells were treated with FF/CAP18 (20, 30 or $40 \mu \mathrm{g} / \mathrm{ml})$. After $48 \mathrm{~h}$, the cells were treated with trypsin and collected in $1.5-\mathrm{ml}$ microtubes. These tubes were centrifuged at $800 \mathrm{x}$ g for $5 \mathrm{~min}$. The supernatant was aspirated and the cell pellets were individually resuspended in $100 \mu \mathrm{l}$ fresh medium and added to $100 \mu \mathrm{l}$ of Muse Annexin V and Dead Cell assay kit reagent. The cellular 
mixture was incubated for $20 \mathrm{~min}$ at room temperature and the cells were examined using a Muse Cell Analyzer (Merck Millipore). These experiments were repeated three times $(n=3)$.

Uptake of exosomes and cell viability analysis. Cell viability was measured by the WST- 8 assay (Dojindo Laboratories). HCT116 cells were seeded $\left(2 \times 10^{3}\right.$ cells/well) in a 96 -well plate with complete culture medium and incubated at $37^{\circ} \mathrm{C}$ in $5 \%$ $\mathrm{CO}_{2}$. After $24 \mathrm{~h}$, exosomes released by FF/CAP18-treated or untreated HCT116 cells were added to the corresponding cells and co-incubated for another $48 \mathrm{~h}$. WST- 8 reagent was added to each well and the samples were incubated for $4 \mathrm{~h}$. Absorbance was measured at a wavelength of $450 \mathrm{~nm}$ using a Synergy ${ }^{\mathrm{TM}}$ HT (BioTek, Winooski, VT, USA). These experiments were repeated three times $(n=3)$. Cell viabilities $(\%)$ were calculated as relative values based on the absorbance of non-treated cells $(100 \%)$.

RNA extraction and miRNA microarray. Exosomes were collected according to the exosome isolation protocol. Total RNA, including miRNAs, was extracted using the miRCURY ${ }^{\mathrm{TM}}$ RNA Isolation Kit-Cell and Plant (Exiqon) according to the appendix protocol of the miRCURY ${ }^{\mathrm{TM}}$ Exosome Isolation Kit-Cells, Urine and CSF (Exiqon). RNA quality [concentration, optical density (OD) 260/280, 260/230 nm] was determined using a BioSpec-nano device (Shimadzu, Kyoto, Japan). The 3D-Gene ${ }^{\mathrm{TM}}$ human microRNA chips for miRNA expression analysis (Toray Industries, Inc., Tokyo, Japan) were used to analyze the effect of FF/CAP18 treatment on miRNA expression in exosomes. Total RNAs labeled with 3D-Gene miRNA labeling kit (Toray Industries, Inc.) were hybridized onto the 3D-Gene Human miRNA Oligo chip (Toray Industries, Inc.). Fluorescent signals were scanned with the 3D-Gene Scanner (Toray Industries, Inc.) and analyzed using 3D-Gene Extraction software (Toray Industries, Inc.). The relative expression level of a given miRNA was calculated by comparing the signal intensities of the valid spots throughout the microarray experiments. The data were globally normalized per array, such that the median of the signal intensity was adjusted to 25. Cellular RNA extraction and miRNA microarray were performed as previously described (12).

Statistical analysis. Data are presented as the mean \pm standard deviation. Statistical significance between two groups was determined by the Student's t-test of variance with least difference post hoc test. $\mathrm{P}<0.05$ was considered to indicate statistically significant differences.

\section{Results}

Characterization of exosomes secreted from HCT116 cells. To ensure successful isolation of exosomes, the collected exosomes from non-treated HCT116 cells were observed as microvesicle clusters (Fig. 1A). For size distribution, 78\% of exosomes were 40-100 nm (Fig. 1B). The amount of exosomes was $3.8 \times 10^{-8}$ and $4.9 \times 10^{-8} \mathrm{mg} / \mathrm{cell}$ in untreated controls and FF/CAP18-treated cells, respectively.

The characterization of exosomes, as determined by western blotting, is shown in Fig. 1C. The investigated CD63 and CD81 proteins were previously reported as being exosome-specific markers $(21,22)$. CD63 was detected at $30-60 \mathrm{kDa}$, as expected from the datasheet for the CD63 antibody. CD81 was detected at $25 \mathrm{kDa}$. These two proteins were detected in HCT116 cells and exosomes before and after treatment with FF/CAP18. In HCT116 cells, the levels of CD63 and CD81 were increased after FF/CAP18 treatment. The expression levels of exosomes from FF/CAP18-treated cells were similar to those in controls, although both proteins were detected.

Localization of FF/CAP18. At $1 \mathrm{~h}$ after treatment with FAM-FF/CAP18, FF/CAP18 interacted with the cell membranes (Fig. 2A). Some FAM-FF/CAP18 was observed in the cells. At $6 \mathrm{~h}$ after treatment, FF/CAP18 was incorporated in the cytoplasm (Fig. 2B). No FF/CAP18 was detected on the cell membrane. As shown in Fig. 2B, the peptide localized in the cytoplasm, but not in the mitochondria or nucleus.

Apoptosis detection using the Annexin V-7-AAD assay. Cells were classified into four groups based on the reactivity of Annexin V and 7-AAD combined, as previously described (23). An increased concentration of FF/CAP18 was accompanied by an increased affinity for Annexin V (Fig. 2C). Treatment with FF/CAP18 at 20 and $30 \mu \mathrm{g} / \mathrm{ml}$ decreased the number of live cells (Annexin $\mathrm{V}^{-}$and 7-AAD) (Fig. 2C, upper right panel and lower left panel). Treatment with FF/CAP18 at $40 \mu \mathrm{g} / \mathrm{ml}$ increased the number of late apoptotic cells and apoptotic cell death (Annexin $\mathrm{V}^{+}$and 7-AAD ${ }^{+}$). To determine the density of FF/CAP18 treatment, HCT116 cells were exposed to FF/CAP18 at a concentration of $33.8 \mu \mathrm{g} / \mathrm{ml}$; this concentration was selected for the experiment as this dose caused the death of $\sim 50 \%$ of HCT116 cells (Fig. 2D).

Viability of HCT116 cells after treatment with exosomes. Exosomes were quantified with the DC protein assay to evaluate the effect of FF/CAP18 on the exosome secretion volume. The amount of pooled exosomes released from cells during FF/CAP18 exposure was $5.0 \times 10^{-8} \mathrm{mg} / \mathrm{cell}$. The amount of exosomes released from non-treated control cells was $3.8 \times 10^{-8} \mathrm{mg}$ per cell. To determine whether exosomes affect the viability of HCT116 cells, exosomes released from control HCT116 cells (Exo CTRL) and from cells treated with FF/CAP18 (Exo FF) were harvested and added to HCT116 cells in the WST- 8 assay. The effect on cell viability was evaluated at exosome protein concentrations of 15.63, 31.25, 62.5, 125 and $250 \mu \mathrm{g} / \mathrm{ml}$. The cell viability (Exo FF group) was significantly decreased at 125 and $250 \mu \mathrm{g} / \mathrm{ml}$ (Fig. 3, $\mathrm{P}<0.01$ ). The effect of Exo CTRL was less prominent compared with that of Exo FF.

miRNA expression profiles of exosomes released by HCT116 cells treated with FF/CAP18. An attempt was made to analyze the alterations in the miRNAs in exosomes to reveal the cause of cell viability suppression. A miRNA microarray revealed that 137 miRNAs were increased by 2 -fold or more in exosomes released by FF/CAP18-treated HCT116 cells compared with exosomes released by non-treated cells (Table I). By contrast, 17 miRNAs were increased by 2-fold or more in HCT116 cells following FF/CAP18 treatment compared with non-treated cells (Table II). Three miRNAs (miR-584-5p, miR-1202 and miR-3162-5p) were upregulated 
A

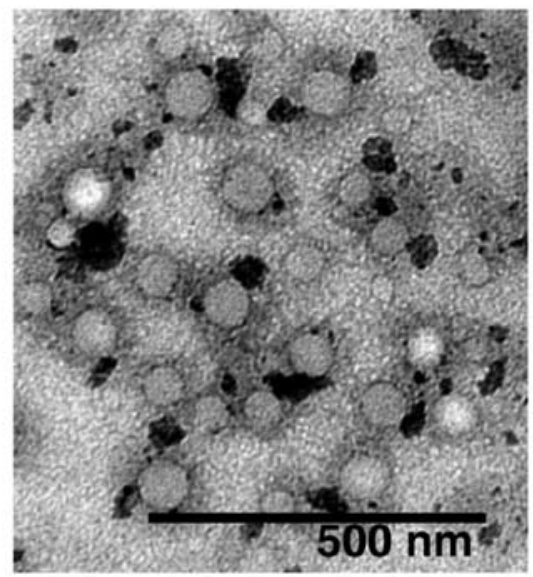

B

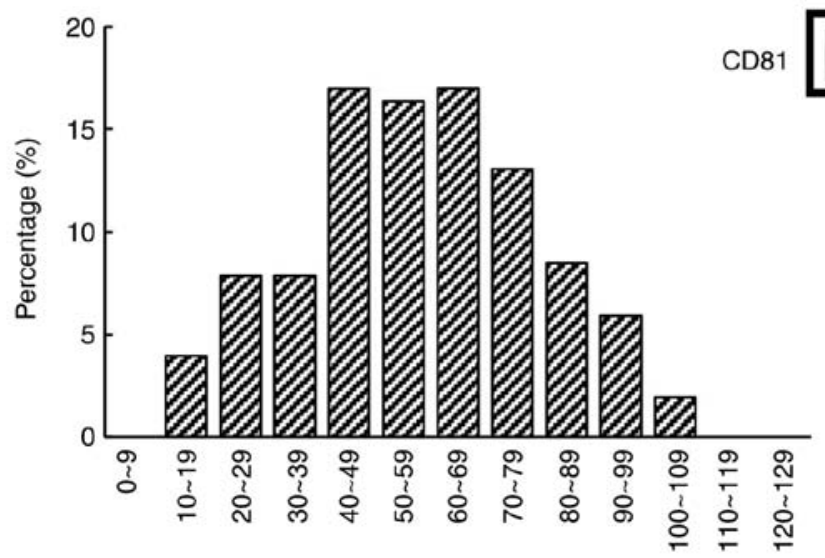

Size of exosomes (nm)
C

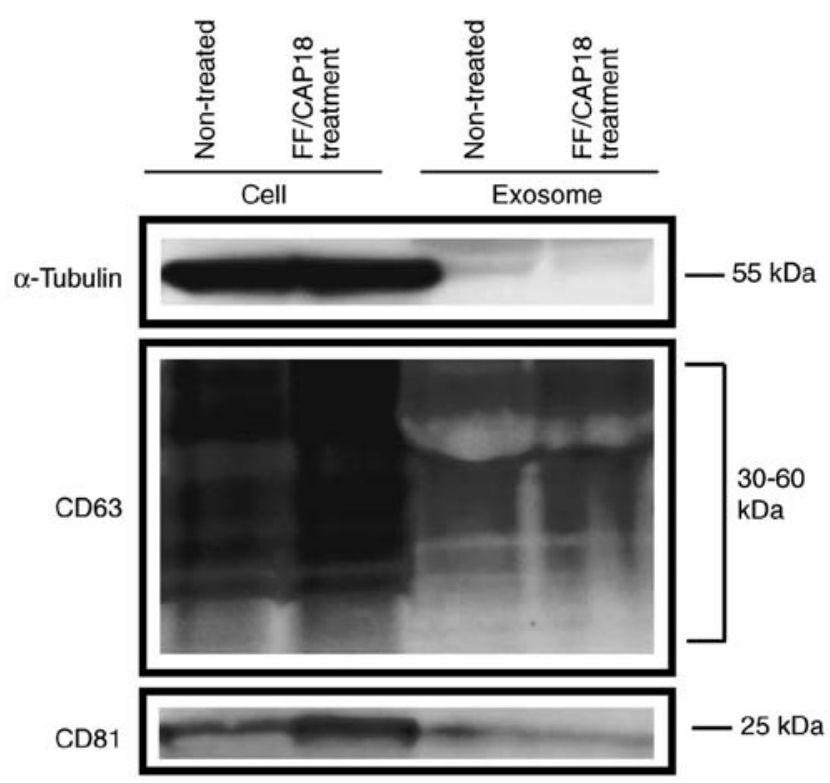

Figure 1. Isolation and characterization of exosomes. (A) Image of exosomes observed by transmission electron microscopy. (B) Size distribution of exosomes secreted by non-treated HCT116 cells. (C) Western blot of $\alpha$-tubulin, CD63 and CD81 in exosomes and cells.

Table I. Upregulated miRNAs in exosomes released from HCT116 cells treated with FF/CAP18.

Fold change

Upregulated miRNAs (miRs) in exosomes

194-3p, 6795-5p, 4485-3p, 378i, 125b-5p, 491-5p, 151b, 31-5p, 500a-3p, 6753-5p, 1273a, 4747-3p, 4689, 765, 1247-5p, 361-5p, 1184, 6861-5p, 493-3p, 1180-3p, 3199, 365a-5p, 3132, 4476, 193b-3p, 502-3p, 378a-3p, 378b, 1910-3p, 769-3p, 6781-3p, 3160-5p, 4291, 375, 1307-5p, 27a-3p, 19a-3p, 4650-3p, 6761-5p, 1202, 93-3p, 4732-5p, 4756-5p, 423-3p, 6722-5p, 320c, 1273e, 92a-3p, 5585-3p, 208a-5p, 4787-3p, 18a-5p, 4804-3p, 4641, 487b-3p, 128-3p, 100-5p, 345-5p, 320a, 3945, 92b-3p, 6774-3p, 151a-3p, 711, 769-5p, 4749-5p, 378g, 7d-3p, 6767-5p, 4450, 4520-5p, 6833-5p, 652-5p, 323a-5p, 584-5p, 6893-5p, 4725-3p, 584-3p, 4472, 18b-5p, 6129, 619-5p, 320b, 3678-3p, 185-5p, 4433b-5p, 8073, 3685, 5088-5p, 151a-5p, 885-3p, 8082, 7854-3p, 28-5p, 130b-3p, 99b-3p, 4669, 634, 106b-5p, 21-3p, 593-5p, 6805-3p, 4306, 320e, 4267

3-4 378d, 4531, 378a-5p, 3162-5p, 6790-3p, 6790-3p, 3619-3p, 659-5p, 6776-5p, 508-5p, 378c, 19b-3p, 4324, 191-5p, 378e, 3616-3p, 3649, 378f, 8078

4-5 4447, 6782-5p, 3126-5p

5-6 4482-3p, 371a-5p, hsa-let-7b-5p, 921

6-7 has-let-7c-5p, 6887-5p

7-8 3687, 15b-5p, hsa-let-7a-5p

$8<\quad$ 4261, hsa-let-7d-5p 
A Phase contrast
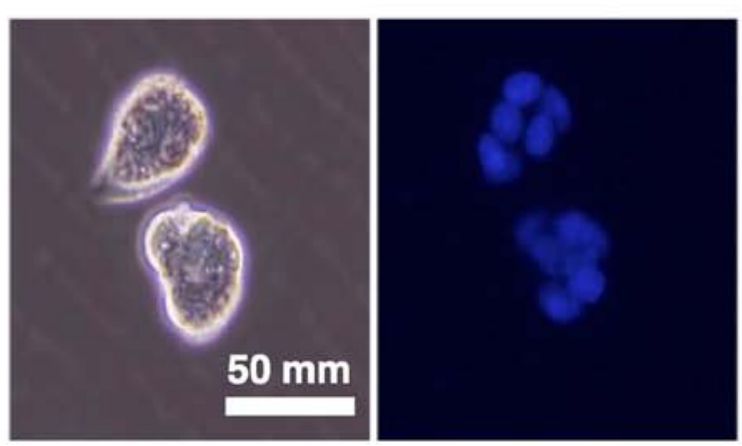

FAM-FF/CAP18

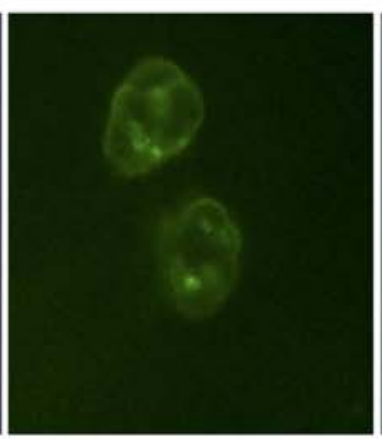

Merge

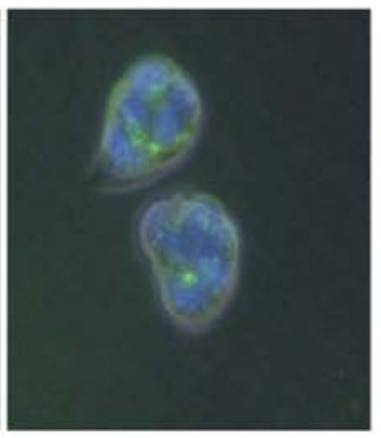

B

Merge

(FAM-FF/CAP18 - DAPI)
Merge

(FAM-FF/CAP18. MitoRed)

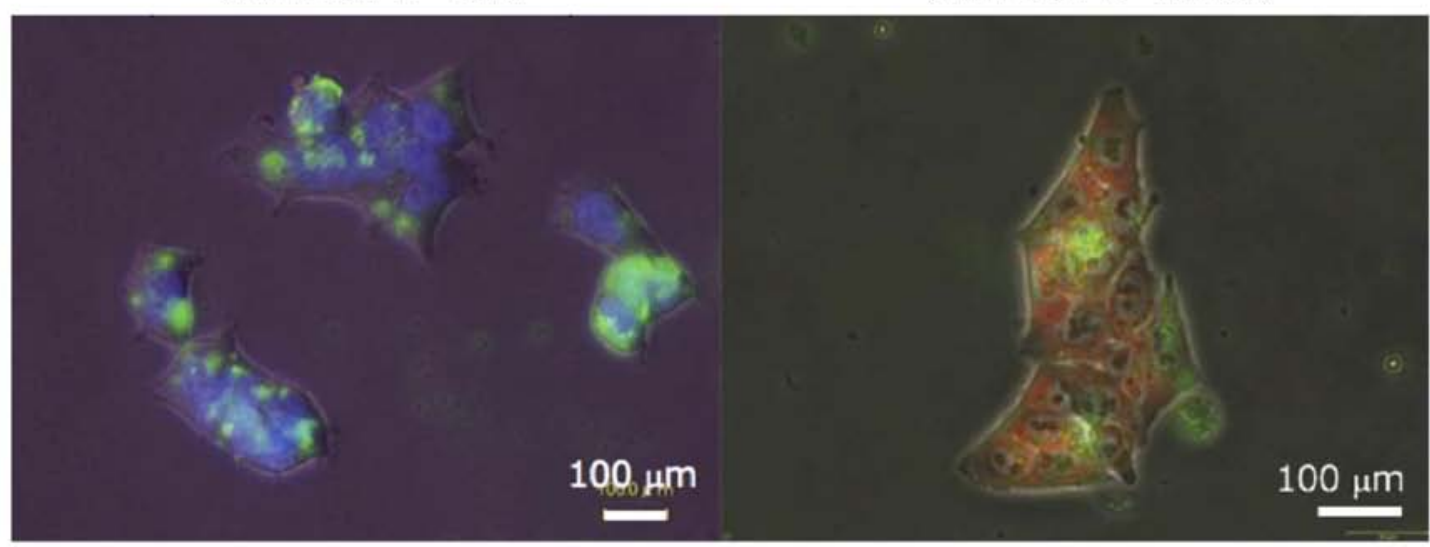

C

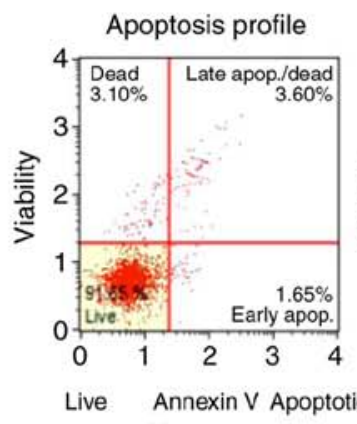

Non-treated
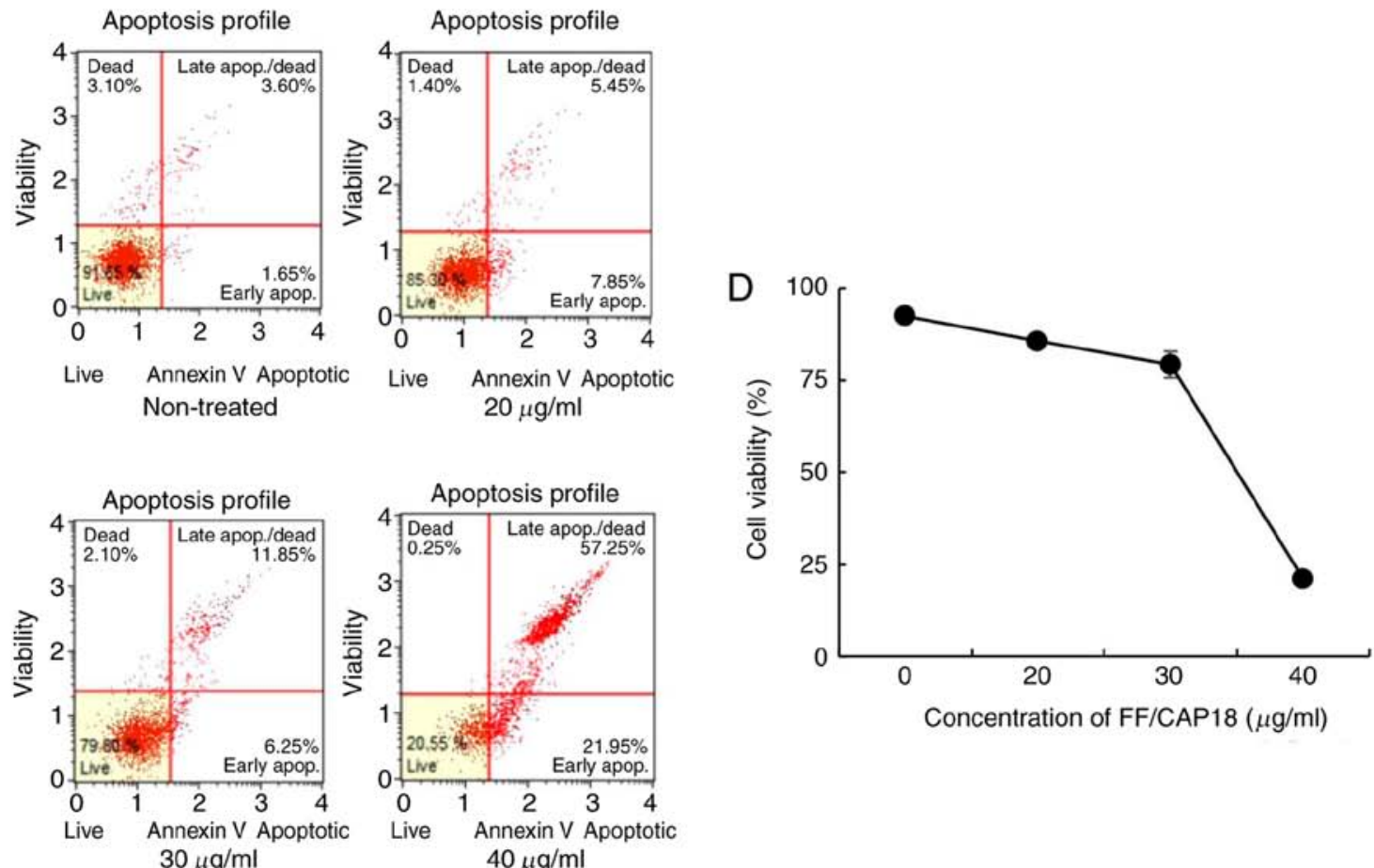

Figure 2. Localization of FF/CAP18 and apoptosis of HCT116 cells after treatment with FF/CAP18. (A) One hour after FAM-FF/CAP18 treatment, HCT116 cells were analyzed by immunofluorescence for FAM-FF/CAP18 (green). DAPI-stained cell nuclei appear blue. (B) Six hours after FAM-FF/CAP18 treatment, HCT116 cells were analyzed by immunofluorescence for FAM-FF/CAP18 (green), with DAPI-stained nuclei again appearing blue. The left panel is s a merged phase contrast image of FAM-FF/CAP18 and DAPI. The right panel is a merged phase contrast image of FAM-FF/CAP18 and MitoRed. (C) Cells were cultured for $48 \mathrm{~h}$ with 20,30, or $40 \mu \mathrm{g} / \mathrm{ml} \mathrm{FF/CAP18}$ and used for the combined Annexin V binding-7-AAD staining assay. Non-treated HCT116 cells (upper left panel) and HCT116 cells treated with FF/CAP18 at concentrations of $20 \mu \mathrm{g} / \mathrm{ml}$ (upper right panel), $30 \mu \mathrm{g} / \mathrm{ml}$ (lower left panel), and $40 \mu \mathrm{g} / \mathrm{ml}$ (right lower panel). (D) Dose response association between HCT116 cell viability (\%) and the concentration of FF/CAP18 (0-40 $\mu \mathrm{g} / \mathrm{ml})$ for $48 \mathrm{~h}$. 


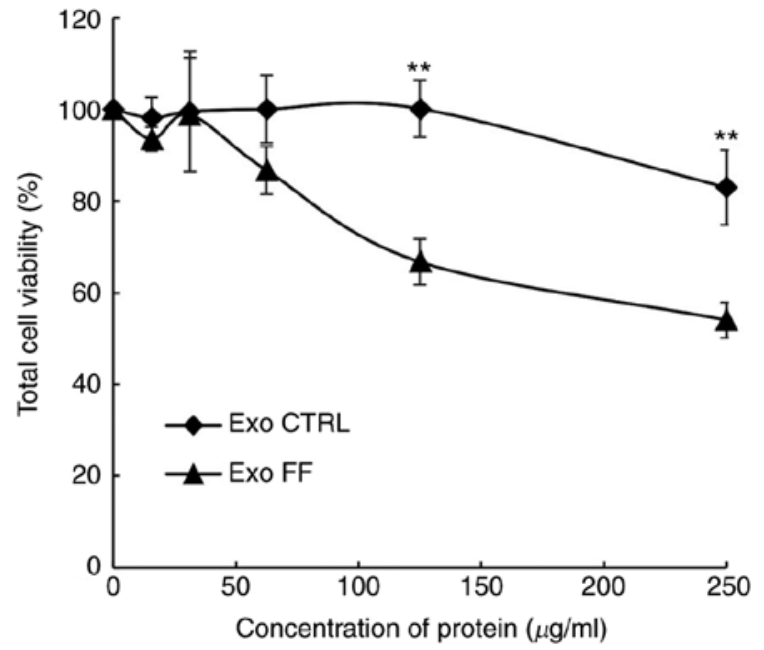

Figure 3. Viability of HCT116 cells treated with exosomes. Exosomes were secreted by both non-treated and FF/CAP18-treated HCT116 cells. ${ }^{* *} \mathrm{P}<0.01$ vs. the control (CTRL) group.

Table II. Upregulated miRNAs in HCT116 cells after treatment with FF/CAP18.

\begin{tabular}{lcc}
\hline $\begin{array}{l}\text { miRNAs } \\
\text { (miRs) }\end{array}$ & $\begin{array}{c}\text { Fold change in } \\
\text { HCT16 after treatment } \\
\text { with FF/CAP18 }\end{array}$ & $\begin{array}{c}\text { More than 2-fold } \\
\text { upregulation } \\
\text { in exosomes }\end{array}$ \\
\hline 4271 & 359 & No \\
575 & 316 & No \\
$483-5 p$ & 287 & No \\
$663 \mathrm{a}$ & 277 & No \\
4298 & 237 & No \\
630 & 195 & No \\
601 & 109 & No \\
$939-5 p$ & 84 & No \\
572 & 71 & No \\
$3663-3 p$ & 58 & No \\
$513 a-5 p$ & 8 & No \\
4257 & 3 & No \\
1202 & 3 & Yes \\
$513 b$ & 3 & No \\
$584-5 p$ & 3 & Yes \\
$3679-5 p$ & 3 & No \\
$3162-5 p$ & 2 & Yes \\
\hline
\end{tabular}

in both HCT116 cells and exosomes following treatment with FF/CAP18.

\section{Discussion}

AMPs play a key role in cancer regulation $(24,25)$. Previous studies revealed the detailed mechanism of the suppression of colon cancer cell growth by the human cathelicidin AMP, LL-37, and its analog, FF/CAP18. This suppression involved the CXCR4-p21 pathway, which is independent of p53, in an miR-663a-targeted manner with an altered metabolic profile $(11,12,23)$. The present study provides insight into the mechanism of cancer growth suppression by AMPs, which occurs via cell-to-cell communication (Fig. 3 and Tables I and II). To the best of our knowledge, the impact of AMPs on tumor cell-to-cell communication has not been previously investigated. In the present study, treatment with FF/CAP18, an analog of LL-37, caused HCT116 cells to secrete more exosomes compared with untreated cells, and these exosomes suppressed HCT116 cell growth. Several miRNAs exhibiting increased expression in cells as well as exosomes following FF/CAP18 treatment were considered to suppress cancer cell growth.

FF/CAP18 became localized on the HCT116 cell membrane, was incorporated into the cells and induced apoptosis (Fig. 2A-C).LL-37 is considered to act by rapidly disrupting the bacterial membrane $(26,27)$. Recently, Shahmiri et al reported that cathelicidin LL-37 exhibits membrane-disrupting antimicrobial activity and two distinct interaction pathways: Pore formation in bilayers of unsaturated phospholipids, and membrane modulation with saturated phospholipids (28). In the present study, membrane disruption was not observed in HCT116 cells following treatment with FF/CAP18. FF/CAP18 was detected on the membrane of HCT116 cells $1 \mathrm{~h}$ after treatment and in the cytoplasm of the cells $6 \mathrm{~h}$ after treatment. These results suggest that, in cancer cells, FF/CAP18 exerted its effects without disrupting the membrane. Additionally, FF/CAP18 treatment of HCT116 cells caused the cells to secrete more exosomes than in the absence of treatment. The secretion of exosomes is regulated by cellular factors, such as intracellular calcium levels, and extracellular factors, such as chemical treatment $(29,30)$. The enhanced exosomal export may be a stress response of HCT116 cells to FF/CAP18. The exosomes released by HCT116 cells during exposure to FF/CAP18 suppressed the viability of HCT116 cells (Fig. 3). This result indicates that exosomes released in the presence of FF/CAP18 contain a tumor suppression factor, such as miR-584-5p, miR-1202 and miR-3162-5p.

The contents of protein and nucleic acids, including miRNAs, of exosomes were previously determined $(13,14)$. miRNAs are crucial for cancer regulation $(18,19)$. FF/CAP18 treatment changed the expression levels of miRNAs in exosomes released by HCT116 cells (Table I). FF/CAP18 treatment induced an increase of 2-fold or more in the expression of three miRNAs (miR-584-5p, miR-1202 and miR-3162-5p) in both HCT116 cells and exosomes, among which miR-584-5p and miR-1202 reportedly act as cancer suppressors. miR-584-5p was reported to inhibit proliferation and induce apoptosis of colon and gastric cancer cells $(31,32)$. miR-584-5p induces apoptotic death by inhibiting the interaction between hnRNP A1 and CDK6 mRNA (32). miR-1202 is downregulated in ovarian cancer and clear cell papillary renal cell carcinoma $(33,34)$. Additionally, miR-1202 suppresses glioma cell proliferation and induces apoptosis by targeting and inhibiting Rab1A (35). miR-584-5p and miR-1202 may suppress the proliferation of HCT116 cells via exosomes. By contrast, miR-3162-5p may be a new regulatory factor in colon cancer. Our group reported that AMPs upregulate the expression of miR-663a in HCT116 cells, and that this miRNA regulates the proliferation of colon cancer HCT116 cells via the CXCR4-p21 pathway (12). However, the expression of miR-663a in exosomes was upregulated by 
$<2$-fold following FF/CAP18 treatment. Therefore, these three miRNAs may exert anticancer effects via exosomes to a greater extent compared with miR-663a. More studies are required to elucidate the mechanism by which these three miRNAs suppress cancer in order to support the use of AMPs as anticancer agents.

The present study focused on the role of FF/CAP18. LL-37 may act in regulating cancer via exosomes. The results of this study indicate that exosomes released by cancer cells in the presence of AMPs suppress tumor growth. Several studies have suggested that exosomes secreted by cancer cells assist in cancer growth and angiogenesis, leading to cancer metastasis $(15,36)$. By contrast, specific exosomes were found to suppress cancer growth. The secretion of miRNAs was mediated through exosomes and their quality and quantity were altered after treatment with FF/CAP18. Alterations in the miRNAs in exosomes released by cells may suppress cancer.

Additionally, LL-37 and FF/CAP18, the analog of the LL-37 peptide, were previously described as anticancer agents $(7,10,11)$. In the present study, FF/CAP18 was also found to inhibit cancer growth through exosomes. Therefore, AMPs act directly on target cancer cells and indirectly via exosomes. The effect of exosomes from HCT116 cells on other type of cancer cells remains unclear. If the exosomes secreted by FF/CAP18-treated HCT116 cells regulate the viability of other types of cancer cells, AMPs may exert anticancer effects against metastatic cancer in other organs.

In recent years, the potential of using exosomes as therapeutic agents, such as exosomes released by mesenchymal stem cells, has attracted attention (37). Studies on exosomes released by cancer cells during treatment with AMPs are important for understanding the therapeutic effects of AMPs against colon cancer.

\section{Acknowledgements}

Not applicable.

\section{Funding}

This study was supported by a Grand-in-Aid for Scientific Research from the Japan Society for the Promotion of Science (2016-2018: Grant number 16H05036).

\section{Availability of data and materials}

All the data generated and analyzed in the present study are available from the corresponding author upon reasonable request.

\section{Authors' contributions}

MH, KK, KI, TI and EI conceived and designed the experiments. $\mathrm{MH}$ and $\mathrm{KK}$ performed the experiments. $\mathrm{MH}$, KK and EI analyzed the date. $\mathrm{MH}$ and EI wrote paper. All the authors have read and approved the final version of the manuscript.

\section{Ethics approval and consent to participate}

Not applicable.

\section{Patient consent for publication}

Not applicable.

\section{Competing interests}

The authors declare that they have no competing interests to disclose.

\section{References}

1. Zanetti M, Gennaro R, Scocchi M and Skerlavaj B: Structure and biology of cathelicidins. Adv Exp Med Biol 479: 203-218, 2000.

2. Zanetti M: The role of cathelicidins in the innate host defenses of mammals. Curr Issues Mol Biol 7: 179-196, 2005.

3. Cowland JB, Johnsen AH and Borregaard N: hCAP-18, a cathelin/pro-bactenecin-like protein of human neutrophil specific granules. FEBS Lett 368: 173-176, 1995.

4. Frohm M, Agerberth B, Ahangari G, Ståhle-Bäckdahl M, Lidén S, Wigzell $\mathrm{H}$ and Gudmundsson GH: The expression of the gene coding for the antibacterial peptide LL-37 is induced in human keratinocytes during inflammatory disorders. J Biol Chem 272: 15258-15263, 1997.

5. Bals R, Wang X, Zasloff M and Wilson JM: The peptide antibiotic LL-37/hCAP-18 is expressed in epithelia of the human lung where it has broad antimicrobial activity at the airway surface. Proc Natl Acad Sci USA 95: 9541-9546, 1998.

6. Hase K, Eckmann L, Leopard JD, Varki N and Kagnoff MF: Cell differentiation is a key determinant of cathelicidin LL-37/human cationic antimicrobial protein 18 expression by human colon epithelium. Infect Immun 70: 953-963, 2002.

7. Ren SX, Cheng AS, To KF, Tong JH, Li MS, Shen J, Wong CC, Zhang L, Chan RL, Wang XJ, et al: Host immune defense peptide LL-37 activates caspase-independent apoptosis and suppresses colon cancer. Cancer Res 72: 6512-6523, 2012.

8. Torre LA, Bray F, Siegel RL, Ferlay J,Lortet-Tieulent J and Jemal A: Global cancer statistics, 2012. CA Cancer J Clin 65: 87-108, 2015.

9. PDQ Adult Treatment Editorial Board: Colon Cancer Treatment $\left(\mathrm{PDQ}^{\circledR}\right)$ : Patient Version. PDQ Cancer Information Summaries. edn. Bethesda (MD): National Cancer Institute (US), 2002.

10. Okumura K, Itoh A, Isogai E, Hirose K, Hosokawa Y, Abiko Y, Shibata T, Hirata M and Isogai H: C-terminal domain of human CAP18 antimicrobial peptide induces apoptosis in oral squamous cell carcinoma SAS-H1 cells. Cancer Lett 212: 185-194, 2004.

11. Kuroda K, Fukuda T, Yoneyama H, Katayama M, Isogai H, Okumura K and Isogai E: Anti-proliferative effect of an analogue of the LL-37 peptide in the colon cancer derived cell line HCT116 p53 $3^{+/+}$and $\mathrm{p} 53^{-/-}$. Oncol Rep 28: 829-834, 2012.

12. Kuroda K, Fukuda T, Krstic-Demonacos M, Demonacos C, Okumura K, Isogai H, Hayashi M, Saito K and Isogai E: miR-663a regulates growth of colon cancer cells, after administration of antimicrobial peptides, by targeting CXCR4-p21 pathway. BMC Cancer 17: 33, 2017.

13. Valadi H, Ekström K, Bossios A, Sjöstrand M, Lee JJ and Lötvall JO: Exosome-mediated transfer of mRNAs and microRNAs is a novel mechanism of genetic exchange between cells. Nat Cell Biol 9: 654-659, 2007.

14. Skog J, Würdinger T, van Rijn S, Meijer DH, Gainche L, Sena-Esteves M, Curry WT Jr, Carter BS, Krichevsky AM and Breakefield XO: Glioblastoma microvesicles transport RNA and proteins that promote tumour growth and provide diagnostic biomarkers. Nat Cell Biol 10: 1470-1476, 2008.

15. Zhou W, Fong MY, Min Y, Somlo G, Liu L, Palomares MR, Yu Y, Chow A, O'Connor ST, Chin AR, et al: Cancer-secreted miR-105 destroys vascular endothelial barriers to promote metastasis. Cancer Cell 25: 501-515, 2014.

16. Ogata-Kawata H, Izumiya M, Kurioka D, Honma Y, Yamada Y, Furuta K, Gunji T, Ohta H, Okamoto H, Sonoda $\mathrm{H}$, et al: Circulating exosomal microRNAs as biomarkers of colon cancer. PLoS One 9: e92921, 2014.

17. Kloosterman WP and Plasterk RH: The diverse functions of microRNAs in animal development and disease. Dev Cell 11: 441-450, 2006.

18. Calin GA and Croce CM: MicroRNA signatures in human cancers. Nat Rev Cancer 6: 857-866, 2006. 
19. Esquela-Kerscher A and Slack FJ: Oncomirs-microRNAs with a role in cancer. Nat Rev Cancer 6: 259-269, 2006.

20. Redis RS, Calin S, Yang Y, You MJ and Calin GA: Cell-to-cell miRNA transfer: From body homeostasis to therapy. Pharmacol Ther 136: 169-174, 2012.

21. Sun Y, Zheng W, Guo Z, Ju Q, Zhu L, Gao J, Zhou L, Liu F, $\mathrm{Xu} Y$, Zhan Q, et al: A novel TP53 pathway influences the HGS-mediated exosome formation in colorectal cancer. Sci Rep 6: 28083, 2016.

22. Zaharie F, Muresan MS, Petrushev B, Berce C, Gafencu GA, Selicean S, Jurj A, Cojocneanu-Petric R, Lisencu CI Pop LA, et al: Exosome-carried microRNA-375 inhibits cell progression and dissemination via Bcl-2 blocking in colon cancer. J Gastrointest Liver Dis 24: 435-443, 2015.

23. Kuroda K, Fukuda T, Isogai H, Okumura K, Krstic-Demonacos M and Isogai E: Antimicrobial peptide FF/CAP18 induces apoptotic cell death in HCT116 colon cancer cells via changes in the metabolic profile. Int J Oncol 46: 1516-1526, 2015.

24. Wu WK, Wang G, Coffelt SB, Betancourt AM, Lee CW, Fan D, $\mathrm{Wu} \mathrm{K}$, Yu J, Sung JJ and Cho CH: Emerging roles of the host defense peptide LL-37 in human cancer and its potential therapeutic applications. Int J Cancer 127: 1741-1747, 2010.

25. Kuroda K, Okumura K, Isogai $H$ and Isogai E: The human cathelicidin antimicrobial peptide LL-37 and mimics are potential anticancer drugs. Front Oncol 5: 144, 2015.

26. Neville F, Cahuzac M, Konovalov O, Ishitsuka Y, Lee KYC, Kuzmenko I, Kale GM and Gidalevitz D: Lipid headgroup discrimination by antimicrobial peptide LL-37: Insight into mechanism of action. Biophys J 90: 1275-1287, 2006.

27. Turner J, Cho Y, Dinh NN, Waring AJ and Lehrer RI: Activities of LL-37, a cathelin-associated antimicrobial peptide of human neutrophils. Antimicrob Agents Chemother 42: 2206-2214, 1998

28. Shahmiri M, Enciso M, Adda CG, Smith BJ, Perugini MA and Mechler A: Membrane core-specific antimicrobial action of cathelicidin LL-37 peptide switches between pore and nanofibre formation. Sci Rep 6: 38184, 2016.

29. Savina A, Furlán M, Vidal M and Colombo MI: Exosome release is regulated by a calcium-dependent mechanism in K562 cells. J Biol Chem 278: 20083-20090, 2003.

30. Safaei R, Larson BJ, Cheng TC, Gibson MA, Otani S, Naerdemann W and Howell SB: Abnormal lysosomal trafficking and enhanced exosomal export of cisplatin in drug-resistant human ovarian carcinoma cells. Mol Cancer Ther 4: 1595-1604, 2005.
31. Li Q, Li Z, Wei S, Wang W, Chen Z, Zhang L, Chen L, Li B, Sun G, $\mathrm{Xu}$ J, et al: Overexpression of miR-584-5p inhibits proliferation and induces apoptosis by targeting WW domain-containing E3 ubiquitin protein ligase 1 in gastric cancer. J Exp Clin Cancer Res 36: 59, 2017.

32. Konishi H, Fujiya M, Ueno N, Moriichi K, Sasajima J, Ikuta K, Tanabe H, Tanaka H and Kohgo Y: microRNA-26a and -584 inhibit the colorectal cancer progression through inhibition of the binding of hnRNP A1-CDK6 mRNA. Biochem Biophys Res Commun 467: 847-852, 2015

33. Nam EJ, Lee M, Yim GW, Kim JH, Kim S, Kim SW and Kim YT: MicroRNA profiling of a CD133 ${ }^{+}$spheroid-forming subpopulation of the OVCAR3 human ovarian cancer cell line. BMC Med Genomics 5: 18, 2012.

34. Munari E, Marchionni L, Chitre A, Hayashi M, Martignoni G, Brunelli M, Gobbo S, Argani P, Allaf M, Hoque MO, et al: Clear cell papillary renal cell carcinoma: micro-RNA expression profiling and comparison with clear cell renal cell carcinoma and papillary renal cell carcinoma. Hum Pathol 45: 1130-1138, 2014.

35. Quan Y, Song Q, Wang J, Zhao L, Lv J and Gong S: MiR-1202 functions as a tumor suppressor in glioma cells by targeting Rab1 A. Tumor Biol 39: 101042831769756, 2017.

36. Meehan K and Vella LJ: The contribution of tumour-derived exosomes to the hallmarks of cancer. Crit Rev Clin Lab Sci 53: 121-131, 2016.

37. Lai RC, Chen TS and Lim SK: Mesenchymal stem cell exosome: A novel stem cell-based therapy for cardiovascular disease. Regen Med 6: 481-492, 2011.

This work is licensed under a Creative Commons Attribution-NonCommercial 4.0 International (CC BY-NC 4.0) License. 